\title{
Un método de cálculo del tamaño muestral en modelos de ecuaciones estructurales
}

CRISTIAN ANTONY RAMOS-VERA*

* Licenciado en Psicología. Universidad César Vallejo, Lima, Perú. E-mail: cristony_777@hotmail.com. 0RCID: 00oo-0oo2-3417-5701. Google Scholar: https://scholar.google.com/citations?hl=en\&user=oyW3ls4AAAAJ. 


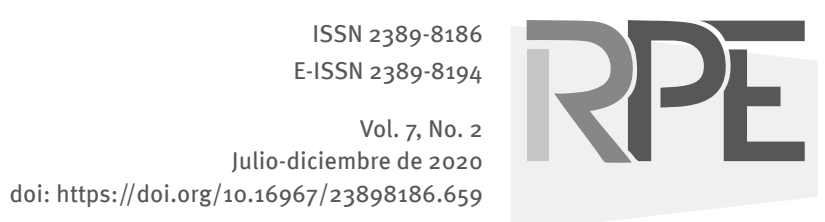

COMO CITAR ESTE ARTÍCULO

How to cite this article:

Ramos-Vera, C. (2020). Un método de cálculo del tamaño muestral en modelos de ecuaciones estructurales. Revista Perspectiva Empresarial, 7(2), 7-9.

\section{Sr. Editor,}

Una estrategia de muestreo es más que necesaria, ya que no siempre es posible reunir datos de cada unidad de la población (Kumar, Talib and Ramayah, 2013). Por tanto, determinar un tamaño apropiado de la muestra es fundamental para tener conclusiones válidas de los resultados de la investigación. No obstante, a menudo, se considera un paso difícil en el diseño de la investigación empírica (Dattalo, 2008).

Aunque hay un buen número de directrices generales para calcular el tamaño de la muestra en diversas áreas de investigación (como, por ejemplo, las ciencias empresariales, marketing, economía y finanzas), muchos investigadores siguen sin tener claro cuál norma deben utilizar para determinar el apropiado tamaño de la muestra en sus estudios; en especial, cuando sus estudios emplean la investigación de encuestas para la recopilación de datos enfocados a los modelos de ecuaciones estructurales - SEM- (Kline, 2016; Kumar, Talib and Ramayah, 2013).

En un estudio de metaanálisis de 74 artículos mediante el enfoque SEM publicados en revistas de alta gestión, Westland (2010) encontró que alrededor del $80 \%$ de todos los estudios se basan en tamaños de muestra insuficientes. De ahí que esta carta tiene como fin presentar un método para estimar el tamaño de muestra en modelos SEM considerando el análisis de potencia estadística, el tamaño del efecto, el nivel de probabilidad, el número de variables latentes y las variables observadas (Soper, 2020).

El método propuesto es útil para cualquier diseño de investigación que emplee una técnica de muestreo probabilístico o no probabilístico para la recogida de datos. Siendo posible calcular la muestra de manera sencilla mediante la calculadora en línea de Soper (2020), la cual permite obtener la cantidad mínima para detectar el efecto en un tamaño de muestra determinado. Este procedimiento sigue la misma lógica que el programa de libre acceso G*Power - que también permite estimar el tamaño de la muestra para estudios de correlación, diferencias de medias y regresiones entre otros-.

Por ejemplo, en un estudio publicado en la presente revista se tuvo en cuenta una muestra de 203 profesionales que participaban en programas de posgrado; en donde se evaluó mediante SEM la influencia de las estrategias de negocio y de las características organizacionales en la innovación técnica (Ostos, Saenz and Bremser, 2019). En el estudio no se menciona el procedimiento para la determinación del tamaño muestral. Utilizando la calculadora online de Soper (2020). En base a doce variables observables y cuatro variables latentes con un tamaño de efecto anticipado de 0,30 , una probabilidad deseada de 0,05 y un 
nivel de potencia estadística de 0,95, el tamaño mínimo recomendado es 207 casos. Por tanto, el estudio anterior utilizó una cantidad de participantes próximo a la cantidad de personas sugeridas.

En conclusión, hoy en día, los métodos para la estimación de un tamaño muestral deben seguir los criterios de potencia estadística o estudios de simulación como el propuesto en la presente carta; y cuya difusión permita generar una mejora en la práctica metodológica para futuros artículos con modelos SEM de la presente revista.

\section{Referencias}

Dattalo, P. (2008). Balancing Power, Precision, and Practicality. New York, USA: Oxford University Press.

Kline, R.B. (2016). Principles and Practice of Structural Equation Modeling. New York, USA: The Guilford Press.

Kumar, M., Talib, S.A. and Ramayah, T. (2013). Business Research Methods. Selangor, Malaysia: Oxford University Press.

Ostos, J., Saenz, A. and Bremser, K. (2019). Relationships of business strategies and organizational characteristics with innovation types: Application in service companies. Revista Perspectiva Empresarial, 6(2), 5-19.

Soper, D.S. (2020). A-priori Sample Size Calculator for Structural Equation Models. Recuperado de http://www.danielsoper.com/statcalc.

Westland, J.C. (2010). Lower bounds on sample size in structural equation modeling. Electronic Commerce Research and Application, 9(6), 476-487. 